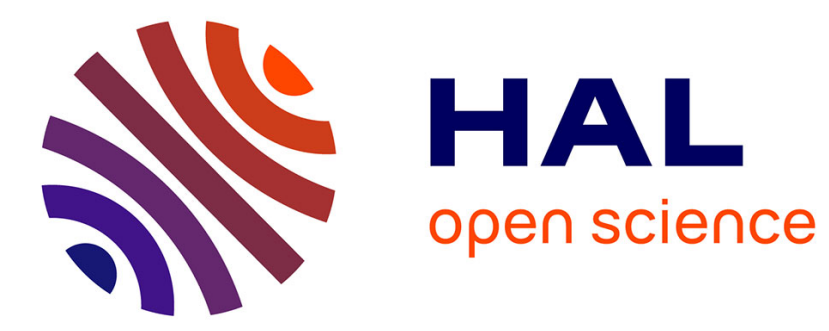

\title{
Equivalence of multistage interconnection networks
}

Jean-Claude Bermond, Jean-Michel Fourneau, Alain Jean-Marie

\section{To cite this version:}

Jean-Claude Bermond, Jean-Michel Fourneau, Alain Jean-Marie. Equivalence of multistage interconnection networks. Information Processing Letters, 1987, 26 (1), pp.45-50. 10.1016/00200190(87)90035-4 . hal-02481509

\section{HAL Id: hal-02481509 \\ https://hal.inria.fr/hal-02481509}

Submitted on 17 Feb 2020

HAL is a multi-disciplinary open access archive for the deposit and dissemination of scientific research documents, whether they are published or not. The documents may come from teaching and research institutions in France or abroad, or from public or private research centers.
L'archive ouverte pluridisciplinaire HAL, est destinée au dépôt et à la diffusion de documents scientifiques de niveau recherche, publiés ou non, émanant des établissements d'enseignement et de recherche français ou étrangers, des laboratoires publics ou privés. 


\title{
Equivalence of Multistage Interconnection Networks
}

\author{
Jean Claude Bermond* \\ Jean Michel Fourneau ** \\ Alain Jean-Marie ** \\ * L.R.I., UA410, C.N.R.S, Informatique, bat 490 \\ Université Paris Sud, 91405 Orsay, FRANCE \\ ** Laboratoire d'Informatique des Systèmes Expérimentaux \\ et de leur Modélisation \\ bat 490, Université Paris Sud, 91405 Orsay, FRANCE
}

\section{ABSTRACT}

In recent years, many multistage interconnection networks using $2 \times 2$ switching cells have been proposed for parallel architecture. We state here a correct and easy graph characterization of all the networks topologically equivalent to the Omega, Flip, Baseline, Reverse Baseline, Indirect Binary Cube, Modified Data Manipulator networks.

Index Terms : Multistage Interconnection Networks, Topological Equivalence, Omega Networks, Connected Components, Directed Graphs. 


\section{1/ Introduction}

Parallel processing techniques use interconnection networks to facilitate concurrent operations and, in fact, the interconnection network is the heart of parallel architecture. Several topologies (designs) have been proposed $[9,10]$, in particular multistage interconnection networks. One of the most investigated scheme consists of a set of $\mathrm{N}=2^{\mathrm{n}}$ inputs (corresponding to the processors), a disjoint set of $\mathrm{N}$ outputs (corresponding to the memories) and $\mathrm{n}$ stages of $2 \times 2$ switching cells. Such networks include the six classical networks : Omega [13], Flip [3], Indirect Binary Cube [15] , Modified Data Manipulator [8], Baseline and Reverse Baseline (see Fig 1)[9]. It is now well known that these networks are related. For instance, Parker has shown [14] that the Flip network and the Indirect Binary Cube network are functionally equivalent, i.e they have the same set of feasible permutations. Wu and Feng [9] proved that these six networks are topologically equivalent. Their methods consisting in exhibiting isomorphisms between two networks do not give a characterization of the equivalence class.

\section{Figure 1}

Another approach consists in modeling the networks by graphs. Such an approach was considered by Agrawal in [1] (see also[2]). He proposed a characterization of this class by "Buddy Properties"; unfortunately, the assertion of Theorem 1 of [1] is false. The aim of this note is to announce a correct characterization. In sections II and III we give precise definitions of the properties used, in terms of graph theory and we show that these properties are not sufficient to insure the equivalence. In section IV we introduce more general properties and give a characterization. Although these properties are easy to check, the proof is too long to be included here; it will appear in[5]. Finally in section $\mathrm{V}$ we show that no local graph properties of this kind are sufficient.

Note that a different approach has been taken by Dias [7] and Kruskal and Snir[12]. They use graph theory plus labelings schemes of the routings in the network. They defined a network isomorphism as a graph isomorphism (they called it topologically equivalent), which furthermore preserves the labels of vertices. They give a nice characterization of the class of networks, called bidelta networks, isomorphic in their sense to the classical ones. Clearly this class is strictly smaller than our's as the authors wanted a stronger property. Our result is different in that sense our characterization use only graph (or topological) properties. 


\section{2/ Graph theoretical approach : MI-digraph}

Interconnection networks can easily be modeled by digraphs in which nodes represent the switching cells and arcs the communication links. Following Agrawal, we also assign nodes to the inputs and the outputs although they do not play any role in the graph properties. For graph theoretical definitions not given here, see for example[6] or any other book on graph theory.

A multistage interconnection digraph (MI-digraph) with $\mathrm{n}$ stages is a digraph with :

$2^{\mathrm{n}}$ special input nodes which are of indegree 0 and outdegree 1 corresponding to the inputs.

$2^{\mathrm{n}}$ special output nodes which are of indegree 1 and outdegree 0 corresponding to the outputs.

All the other nodes ( corresponding to switching cells) are of indegree 2 and outdegree 2. These nodes are partitioned into $\mathrm{n}$ ordered stages; each stage consisting of independent nodes. There are arcs only from input nodes to the first stage, from nodes of the last stage to output nodes, and from nodes of the $i^{\text {th }}$ stage to nodes of the $(i+1)^{\text {th }}$ stage.

With this definition we will say that two multistage interconnection networks are topologically equivalent if their MI-digraphs are isomorphic. Two digraphs are isomorphic if there exists a bijection from the nodes of the first digraph into the nodes of the second one, which preserves the relationship of adjacency. We will denote by $B_{k}$ the $k$-stage Baseline MI-digraph.

Remark : In all the figures, the arcs are directed from left to right.

\section{3/ MI-digraphs Properties :}

\section{Banyan Property}

One minimal requirement is to allow a connection between any pair of input and output nodes. We will say that a digraph has the Banyan Property if and only if for any input node and any output node there exists a unique path connecting them. For MI-digraph the existence of such paths is sufficient; uniqueness is a consequence of the definition.

Another property common to the six canonical networks was considered in [1] and[2] where two slightly different variants were considered.

Buddy Property : An MI-digraph has the Buddy Property if when two nodes of an arbitrary stage have a common son, then their two sons are identical.

Remark : Two nodes of a same stage have therefore either no common sons or exactly the same two sons. The connected components of the digraph formed by the nodes of two consecutive stages are 
disjoint cycles of length 4 .

These properties are too weak to imply the topological equivalence. Let us consider the MI-digraph described in Figure 2. One can easily check that it satisfies the Banyan Property and the Buddy Property. However it is not isomorphic to the Reverse Baseline MI-digraph drawn in Figure 1. Indeed this MI-digraph has cycle of length 10 (the bold line in Figure 2), but the Reverse Baseline digraph has no such cycle. Another counterexample is described in [2].

Figure 2

Strict Buddy Property : Following Agrawal [1] we say that an MI-digraph has the Strict Buddy Property if it satisfies the Buddy Property and if for any nodes $j_{1} j_{2} j_{3} j_{4}$ such that $: j_{1}, j_{2}$ have the same two fathers; $j_{3}, j_{4}$ have the same two fathers; $j_{1}, j_{3}$ have the same two sons; then $j_{2}, j_{4}$ have the same two sons (see Figure 3).

Agrawal claimed in [1] that all the MI-digraphs with $\mathbf{n}$ stages satisfying the Banyan Property and the Strict Buddy Property are isomorphic. Unfortunately that is true only if $\mathrm{n} \leq 4$

Figure 3

Proposition : If $n \geq 5$, there exist MI-digraphs satisfying the Strict Buddy Property which are not isomorphic.

Proof : Consider the example of Figure 4. This network satisfies the Banyan Property and the Strict Buddy Property, but it is not isomorphic to the Baseline network. Indeed the graph obtained by removing the vertices of the last stage of this graph is still connected but the same operation on a Reverse Baseline network disconnects the graph. This construction can easily be extended for $n>5$. 


\section{4/ Characterization}

Connected Components of an MI-digraph are those of the undirected underlying graph obtained from the digraph by deleting the orientation.

$\mathbf{P}(\mathrm{i}, \mathrm{j})$ Property : We will say that an MI-digraph with n stages has the property $P(i, j)$ for $1 \leq i \leq j \leq n$ if the subdigraph $(G)_{i, j}$ induced by the vertices of the stages from $i$ to $j$, has exactly $2^{n-1-(j-i)}$ connected components.

Remark : $P(i, i)$ means that the $i-t h$ stage has $2^{n-1}$ nodes. Similarly, $P(i, i+1)$ means that $(G)_{i, i+1}$ has $2^{n-2}$ components; so any component has at least 4 vertices and as there are $2^{\text {n }}$ vertices in stage $i$ and $i+1$, every component has exactly 4 vertices. Thus $P(i, i+1)$ is equivalent to the Buddy Property. Similarly one can verify that the Strict Buddy Property is equivalent to the conjunction of $\mathrm{P}(\mathrm{i}, \mathrm{i}+1)$ and $\mathrm{P}(\mathrm{i}, \mathrm{i}+2)$.

Property $\mathbf{P}\left({ }^{*},{ }^{*}\right)$ : We will say that an MI-digraph has property $\mathrm{P}\left({ }^{*},{ }^{*}\right)$ if and only if it satisfies $\mathrm{P}(\mathrm{i}, \mathrm{j})$ for every ordered pair $i, j$ such that $1 \leq i \leq j \leq n$

One can show that the 6 canonical MI-digraphs satisfy $\mathrm{P}\left({ }^{*}, *\right)$. But the MI-digraph of Figure 2 does not satisfy $\mathrm{P}(1,3)$. Indeed the subdigraph consisting of the vertices of the 3 first stages has only one component. This is another proof that it is non isomorphic to the Reverse Baseline MI-digraph. Similarly the MI-digraph of Figure 4 does not satisfy $\mathrm{P}(1,4)$ and therefore cannot be isomorphic to the Reverse Baseline MI-digraph. In summary $\mathrm{P}\left({ }^{*}, *\right)$ and the Banyan Property are necessary conditions for the equivalence with the Reverse Baseline. The following theorem, whose proof can be found in [4] and[5], shows they are also sufficient.

Theorem : All the MI-digraphs with $\mathrm{n}$ stages satisfying the Banyan Property and $\mathrm{P}\left({ }^{*},{ }^{*}\right)$ are isomorphic.

$\mathrm{P}\left({ }^{*}, *\right)$ is a strong graphical property which might be difficult to check. The following lemma of $[4,5]$ shows that one can limit the number of verifications to $2 n$ elementary properties and therefore the complexity of checking the property is linear in the number of vertices.

Lemma An MI-digraph, which satisfies the Banyan Property, satisfies $\mathrm{P}\left({ }^{*},{ }^{*}\right)$ if and only if it satisfies $\mathrm{P}\left(1,{ }^{*}\right)$ (that is $\mathrm{P}(1, \mathrm{j})$ for $\left.1 \leq \mathrm{j} \leq \mathrm{n}\right)$ and $\mathrm{P}\left({ }^{*}, \mathrm{n}\right)$ (that is $\mathrm{P}(\mathrm{i}, \mathrm{n})$ for $\left.1 \leq \mathrm{i} \leq \mathrm{n}\right)$.

Remark : in $[4,5]$ it is also shown that for an MI-digraph belonging to this equivalence class, the connected components $(G)_{i, i+k}$ are all isomorphic to $B_{k}$ the k-stage Baseline MI-digraph. 


\section{5/ Lack of local characterization in term of connected components}

We have described graphs proving that, for a Banyan MI-digraph, neither P(i,i+1) (Buddy Property) nor $\mathrm{P}(\mathrm{i}, \mathrm{i}+1)$ and $\mathrm{P}(\mathrm{i}, \mathrm{i}+2)$ (Strict Buddy Property) alone could imply topological equivalence with the Baseline MI-digraph.

Nevertheless, it is easy to check, using the last lemma, that if the digraph is Banyan and satisfies: $\mathrm{P}(\mathrm{i}, \mathrm{i}+\mathrm{k}), \forall \mathrm{k} \leq \mathrm{n}-2$, then it satisfies $\mathrm{P}\left(1,{ }^{*}\right)$ and $\mathrm{P}\left({ }^{*}, \mathrm{n}\right)$, and therefore is equivalent to $\mathrm{B}_{\mathrm{n}}$.

As stated in the following Theorem, there is no weaker property that can insure topological equivalence of Banyan MI-digraphs.

Theorem For $n=>k+3$, there exist non isomorphic MI-digraphs satisfying the properties $P(i, i+j)$ for all $\mathrm{j} \leq \mathrm{k}$ and $\mathrm{i} \leq \mathrm{n}-\mathrm{j}$.

Outline of Proof : We prove this theorem (for $\mathrm{n}>\mathrm{k}+3$ ), by constructing a Banyan MI-digraph, $\mathrm{G}$, satisfying $P(i, i+k)$, for every $k<n-2$, which is not isomorphic to $B_{n}(F i g)$ ).

$\Rightarrow \quad(G)_{1, n-2}$ is constituted of four $B_{n-2}$ superposed.

$\Rightarrow \quad$ Let us denote a vertex $x$ of the $(n-2)$ th row of $G$ by its binary decomposition $\left(x_{n-1}, x_{n-2}, \cdots, x_{1}\right)$ and let $\bar{x}=\left(x_{n-4}, x_{n-5}, \cdots, x_{1}\right)$ and $\hat{x}=\left(x_{n-3}, x_{n-4}, \cdots, x_{1}\right)$.

$$
x=\left(x_{n-1}, x_{n-2}, \cdots, x_{1}\right)=\left(x_{n-1}, x_{n-2}, x_{n-3}, \bar{x}\right)=\left(x_{n-1}, x_{n-2}, \hat{x}\right)
$$

The nodes $(0,0,0, \bar{x})$ and $(0,1,0, \bar{x})$ of the $n-2$ th row are connected to the nodes $(0,0, \bar{x}, 0)$ and $(0,0, \bar{x}, 1)$ of the $n-1$ th row,

The nodes $(0,0,1, \bar{x})$ and $(1,0,0, \bar{x})$ of the $n-2$ th row are connected to the nodes $(0,1, \bar{x}, 0)$ and $(0,1, \bar{x}, 1)$ of the $n-1$ th row,

The nodes $(0,1,1, \bar{x})$ and $(1,1,0, \bar{x})$ of the $n-2$ th row are connected to the nodes $(1,0, \bar{x}, 0)$ and $(1,0, \bar{x}, 1)$ of the $\mathrm{n}-1$ th row,

The nodes $(1,0,1, \bar{x})$ and $(1,1,1, \bar{x})$ of the $n-2$ th row are connected to the nodes $(1,1, \bar{x}, 0)$ and $(1,1, \bar{x}, 1)$ of the $n-1$ th row.

$\Rightarrow$ The nodes $(0,0, \hat{x})$ and $(1,1, \hat{x})$ of the $n-1$ th row are connected to the nodes $(0, \hat{x}, 0)$ and $(0, \hat{x}, 1)$ of the last row,

The nodes $(0,1, \hat{\mathrm{x}})$ and $(1,0, \hat{\mathrm{x}})$ of the $\mathrm{n}-1$ th row are connected to the nodes $(1, \hat{\mathrm{x}}, 0)$ and $(1, \hat{\mathrm{x}}, 1)$ of the last row. 
First, one can easily verify that $G$ is Banyan. Next, it is easy, but somewhat tedious to verify that $G$ satisfies $P(i, i+k), \forall k \leq n-3$. Finally, we observe that $G$ is not isomorphic to $B_{n}$, because $(G)_{1, n-1}$ is connected, while $\left(B_{n}\right)_{1, n-1}$ has two connected components. The key idea in this construction was to connect as early as possible, scanning the graph from left to right, 4 connected components instead of 2 . The point is that it is then still possible to complete the last column in order to obtain a Banyan graph.

\section{Conclusion :}

We hope to have clarified the graph characterization of multistage interconnection networks. note that all the results can be extended to $\mathrm{KxK}$ switching cells (i.e. the internal nodes of the MII digraph being of outdegree and indegree $\mathrm{k}$, see for details[11]). Using our theorem it is now easy to see if a new network is equivalent to a classical one. Furthermore the theorem can be used to show that a family of networks defined by some algebraic properties is in the equivalence class of the six classical ones (see [11]). This class seems to be the most interesting, because it is in some sense the most regular. Therefore, inside this class the designer can choose a network according to other criteria : permutations which are realized by the network, routing algorithms... For example if one wants to have a simple digit controlled routing algorithm using only the addresses of the processors, one can choose the interesting subclass consisting of bidelta networks (see $[7,12]$ ).

Acknowledgment : we thank the referee for his valuable remarks and for drawing our attention to the results of Dias, Kruskal and Snir. 


\section{References}

1. Agrawal, D.P., "Graph theoretical analysis and design of multistage interconnection networks," IEEE Trans. Computers, vol. C32, pp. 637-648, Jul. 1983.

2. Agrawal, D.P. and Kim, S.C., "On non-equivalent multistage interconnection networks," Proc. Int. Conf. Parallel Processing, pp. 234-237, 1981.

3. Batcher, K.E., "The flip network in Staran," Proc. Int. Conf. Parallel Processing, pp. 65-71, Aug 1976.

4. Bermond, J.C, Fourneau, J.M, and Jean-Marie, A., "Equivalence topologique des réseaux d'interconnexion multi-étages," Rapport ISEM 28, Mar. 1985.

5. Bermond, J.C., Fourneau, J.M., and Jean-Marie, A., "A graph theoretical approach to equivalence of multistage interconnection networks," Rapport LRI 242, Nov 1985. submited to Discrete Applied Math.

6. Bondy, J.A. and Murty, U., Graph theory with applications, MacMillan Press, 1976.

7. Dias, D.M., "Packet communication in delta and related networks," Ph.D. thesis Rice University, 1981.

8. Feng, T., "Data manipulating functions in parallel processors and their implementations," IEEE Trans. Computers, vol. C23, pp. 309-318, Mar. 1974.

9. Feng, T. and Wu, C., "On a class of multistage interconnection networks," IEEE Trans. Computers, vol. C29, pp. 694-702, Aug. 1980.

10. Feng, $\mathrm{T}$. and $\mathrm{Wu}, \mathrm{C} .$, Tutorial : Interconnection networks for parallel and distributed processing, IEEE Publications, 1984.

11. Fourneau, J.M., "Modélisation des réseaux d'interconnexion," Thèse de troisième cycle, Univ. Paris Sud, 1985.

12. Kruskal, C. P. and Snir, M., "A unified theory of interconnection network structure," To appear in T.C.S., 1986.

13. Lawrie, D.H., "Access and alignment of data in an A.P.," IEEE Trans. Computers, vol. C24, pp. 1145-1155, Dec. 1975.

14. Parker, D.S., "Notes on shuffle/exchange type networks," IEEE Trans. Computers, vol. C29, pp. 213-222, Mar. 1980.

15. Pease, M.C., "The indirect binary cube microprocessors array," IEEE Trans. Computers, vol. C26, pp. 458-473, May 1977. 

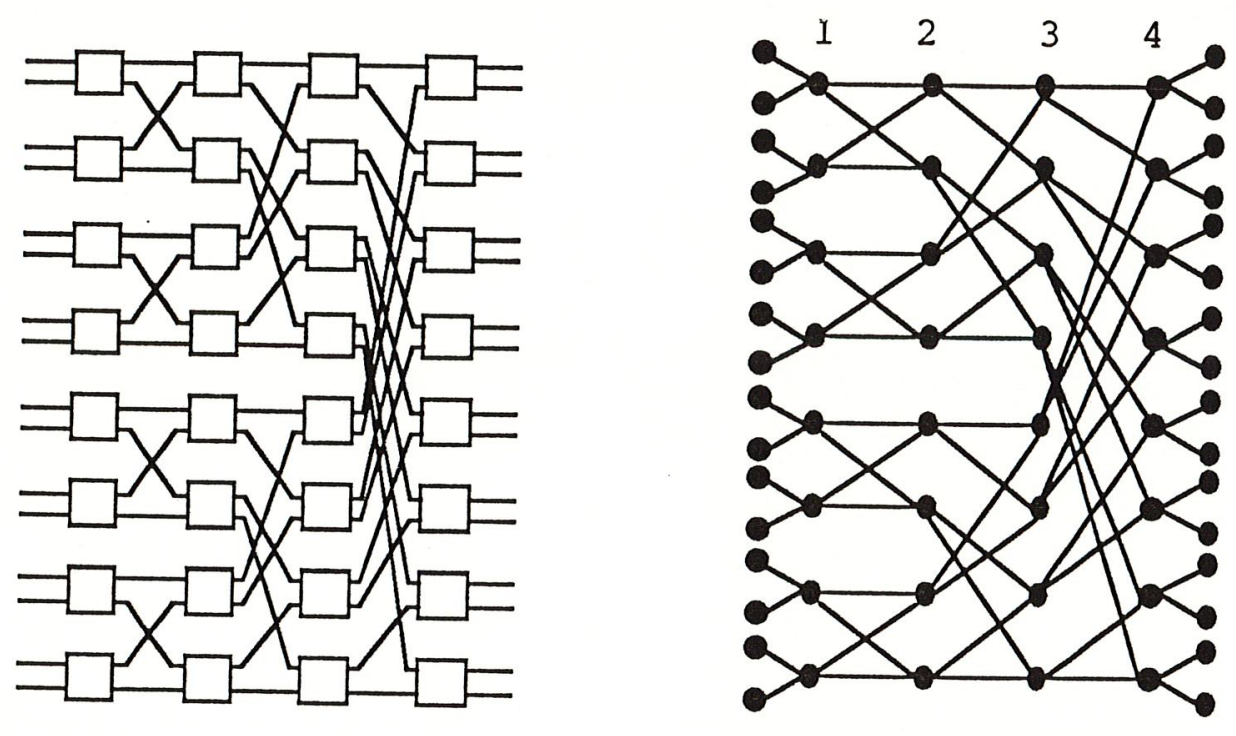

Figure 1 : Reverse Baseline Network and Digraph

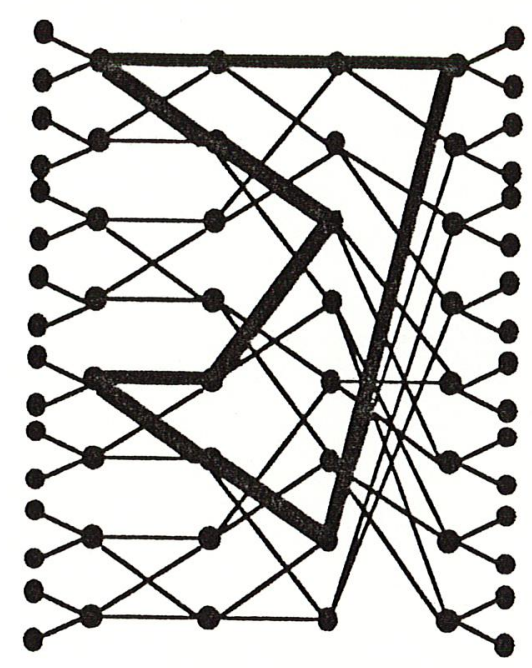

Figure 2: A non isomorphic MI-digraph

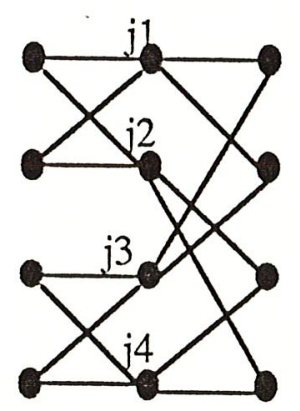

Figure 3 : Strict Buddy Property 


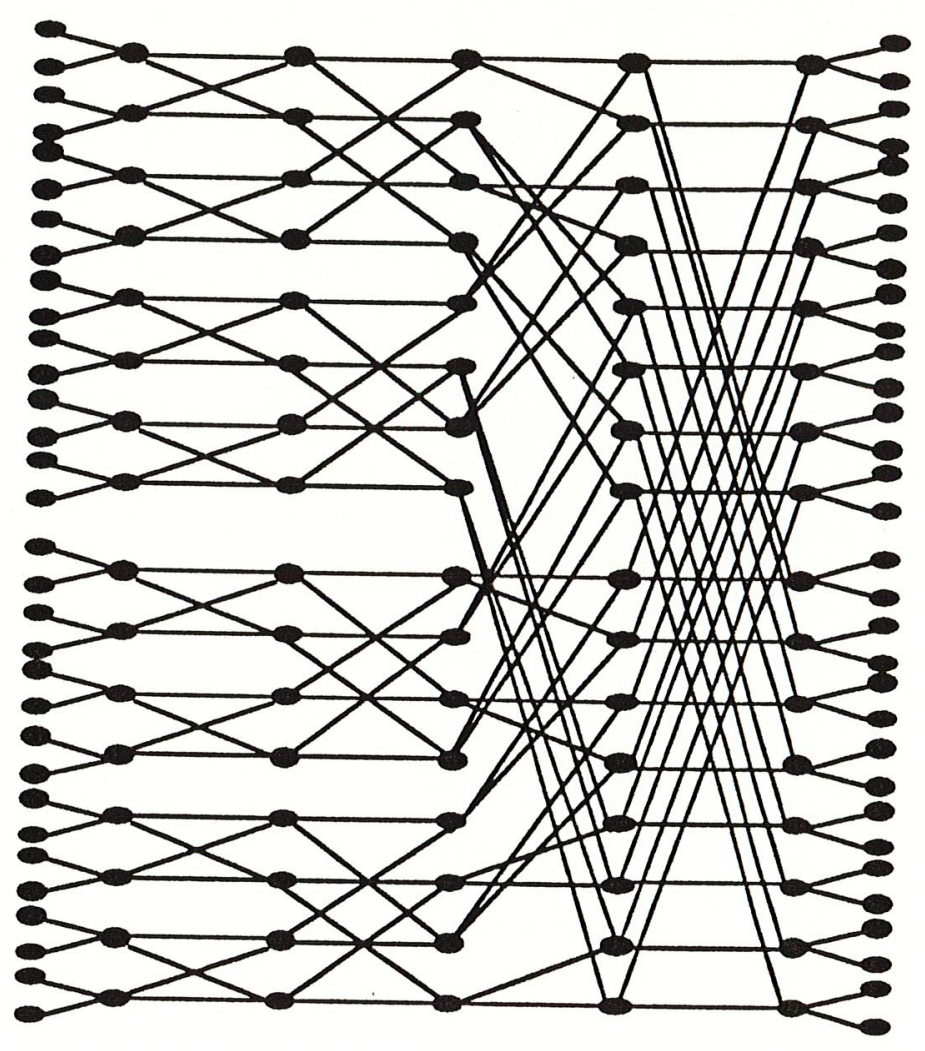

Figure 4 : A non isomorphic MI-digraph satisfiyng the Strict Buddy Property

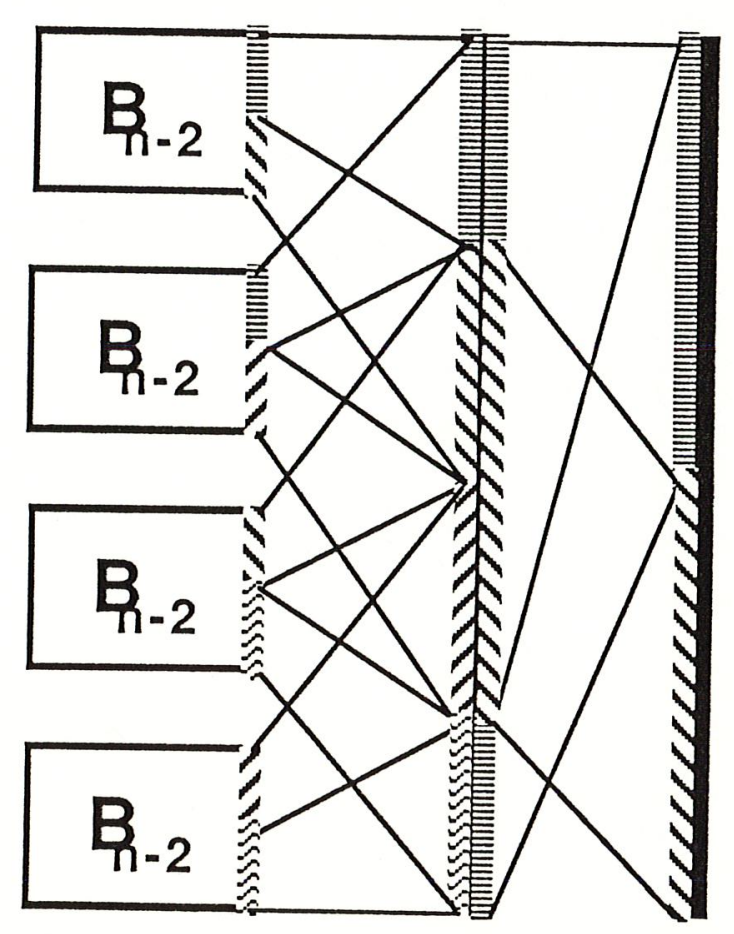

Figure 5 : 